\title{
Role of cardiovascular magnetic resonance in acute and chronic ischemic heart disease
}

\author{
${\text { A. } \text { Baritussio }^{1} \cdot \text { A. Scatteia }}^{1} \cdot$ C. Bucciarelli-Ducci ${ }^{1}$
}

Received: 21 February 2017 / Accepted: 9 March 2017 / Published online: 18 March 2017

(C) The Author(s) 2017. This article is an open access publication

\begin{abstract}
Cardiovascular magnetic resonance (CMR) is a multi-parametric, multi-planar, non-invasive imaging technique, which allows accurate determination of biventricular function and precise myocardial tissue characterization in a one-stop-shop technique, free from the use of ionizing radiations. Though CMR has been increasingly applied over the last two decades in every-day clinical practice, its widest application has been in the assessment of ischemic cardiomyopathy.
\end{abstract}

Keywords Cardiovascular magnetic resonance $\cdot$ Acute myocardial infarction $\cdot$ Chronic ischemic heart disease

\section{Acute myocardial infarction}

Coronary artery disease (CAD) is the leading cause of death worldwide [1]. Although the incidence rate of STelevation myocardial infarction (STEMI) has declined, that of non-ST elevation myocardial infarction (NSTEMI) has increased over the last few decades [1]. Diagnosis usually relies on clinical history and electrocardiographic changes, while imaging, mainly trans-thoracic echocardiography (TTE), is usually deferred.

Acute coronary occlusion determines cytogenic and vasogenic myocardial oedema, which is a rather non-specific response to an acute insult (ischemic, inflammatory, traumatic), and is characterized by an increase in water

C. Bucciarelli-Ducci

c.bucciarelli-ducci@bristol.ac.uk

1 Bristol Heart Institute, Bristol NIHR Cardiovascular Biomedical Research Unit (BRU), Upper Maudlin Street, Bristol BS2 8HW, UK content [2]. CMR has the ability to distinguish fat, fluid and soft tissue by using different imaging protocols that exploit different intrinsic tissue properties [3, 4]. The linear correlation between myocardial water content and $\mathrm{T} 2$ transverse relaxation time in acute myocardial infarction was first described in the early 80s; T2-weighted sequences were subsequently developed and implemented in clinical practice to assess the presence of myocardial oedema, which appears as an area of increased signal intensity (bright). Pathophysiologic model of acute ischemia after coronary occlusion shows a rapid onset of myocardial oedema, which can be detected as early as $30 \mathrm{~min}$ from symptoms onset on T2-weighted images (Fig. 1a) [5]. The territory of distribution of the infarct-related artery is at risk of potentially irreversible damage (myocardium at risk) if reperfusion does not occur promptly [6]. When myocardial blood flow is promptly restored, the irreversibly damaged myocardial area is significantly smaller than the area at risk. T2-weighted sequences can detect the presence and extent of the myocardium at risk and derive the amount of myocardial salvage by subtracting the extent of scarred myocardium in the post-contrast sequences (late gadolinium enhancement, LGE) (Fig. 1b) from the area at risk on T2-weighted images. T2-weighted sequences for the assessment of the area at risk have been validated in animal studies versus the gold standard fluorescent microspheres, showing comparable findings [7]. The assessment of salvage myocardium is reproducible and has been validated against SPECT and angiographic studies [5]; moreover, while SPECT tracer needs to be given prior to revascularization, CMR provides the opportunity to assess salvage myocardium retrospectively, few days after the acute event, without interfering with the acute clinical management [8]. Identification of myocardial oedema in a patient with suspected acute coronary syndrome (ACS) not only confirms 
Fig. 1 Myocardial infarction: overview. T2-weighted two chamber long axis view showing myocardial oedema in the basal to mid-cavity inferior wall (a) with concomitant subendocardial late gadolinium enhancement (LGE) in the post-contrast sequence (b) in a patient with acute subendocardial infarction in the proximal to mid right coronary artery territory. T2-weighted three chamber long axis view showing myocardial oedema in the mid-apical anteroseptum with evidence of a hypo-intense core (c, white arrow), consistent with intramyocardial haemorrhage, in a patient with transmural infarction in the distal left anterior descending territory (d) with persistence of microvascular obstruction (d, white arrow). Four chamber long axis post-contrast view showing subendocardial LGE of the mid-cavity anterolateral wall (e). Two chamber long axis post-contrast view showing transmural LGE of the midapical inferior wall (f)
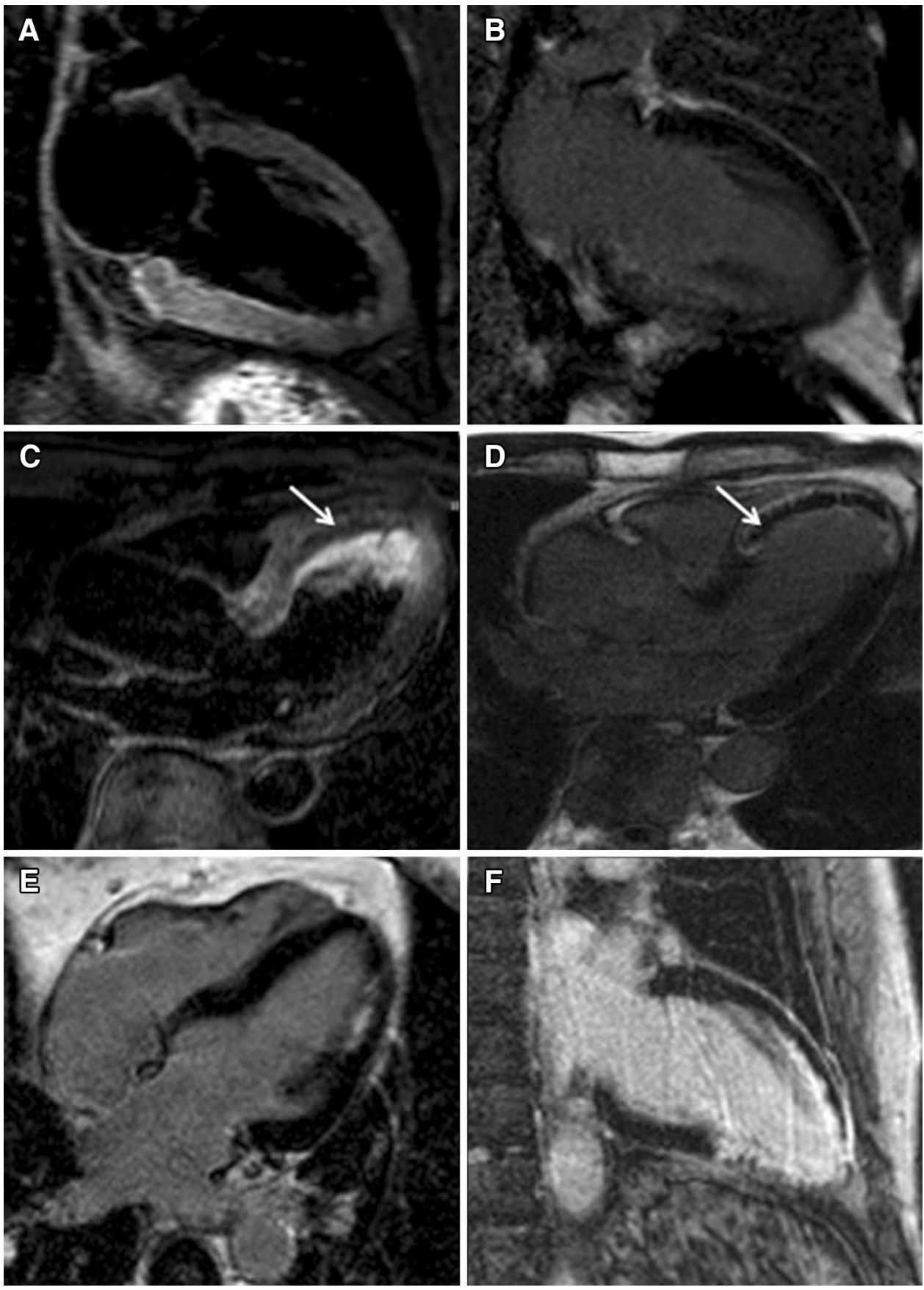

the diagnosis, but also helps in establishing the age of the myocardial infarction [9]. All patients presenting with ACS and those with high pre-test probability of CAD undergo coronary angiography in keeping with existing guidelines. However, in a non-negligible proportion of patients with ACS (approximately one-third), a "culprit" lesion is not identified by angiogram [10]; these cases are often attributed to undetectable coronary pathology, such as distal embolization, coronary spasm and myocardial bridging. According to the ischemic wave-front phenomenon, myocardial oedema extends from the subendocardium to the entire wall thickness of the territory supplied by the occluded vessel. Based on this pathophysiologic model, T2-weighted sequences can easily allow the detection of the culprit lesion, as an area of increased signal intensity along the distribution territory of a coronary artery (Fig. 1a). Monney et al. [11] found that myocardial infarction was identified in $13 \%$ of patients with ACS and unobstructed coronaries, as delineated by a localised increase in signal intensity on T2-weighted images in areas of transmural scar. Though persistence of myocardial oedema after an acute event varies according to different studies, infarct 
related oedema can persist from few weeks to few months after the event, so that it can also be detected once cardiac biomarkers have normalized [5]. As the abnormalities are transient, imaging these patients within 2 weeks from the event represents an optimal time window before the abnormalities resolve and become no longer detectable [12]. Not all patients in the NSTEMI-ACS population have evidence of CAD requiring revascularization; Raman et al. [13] showed that the presence of myocardium at risk on T2-weighted CMR sequences allows the identification of patients who will benefit from an early invasive management. In fact, the presence of myocardial oedema has been shown to increase the risk of adverse cardiovascular events, irrespective of revascularization [14]. Clinical evidence has shown that prompt myocardial revascularization reduces the infarcted area and the incidence of long-term adverse events. However, myocardial revascularization can itself also lead to myocardial damage, the so called "ischemia-reperfusion injury", as shown by histology studies soon after revascularization. Prolonged ischemiainduced microvascular damage leads to absent distal myocardial flow (no-reflow phenomenon) on coronary angiography, which can be identified as either microvascular obstruction or intra-myocardial haemorrhage (IMH) on CMR. Both entities are a consequence of microvascular cell necrosis and present in the context of a large and late reperfused myocardial infarction [15]. Cardiovascular magnetic resonance soon after an acute ischemic event is able to detect the ischemia-reperfusion injury (Fig. 1c, d). The imaging equivalent of the angiographic and histologic noreflow phenomenon is known as microvascular obstruction (MVO) and appears as a non-perfused, non-contrast gaining area within the infarcted myocardium (Fig. 1d); this can be detected after contrast administration and has typically low signal intensity (black) both in the early and late acquisition (5 and 15 min after contrast administration, respectively). As contrast agent needs time to diffuse into the nonperfused area, MVO extent also depends on time to image acquisition, appearing larger early after contrast administration and smaller when the images are acquired later as the contrast ultimately fills in these areas [16]. Extensive late MVO thus represents more severe microvascular damage [8]. Rochitte et al. [17] have shown that there is a timecourse of MVO development, with onset within the first $3.5 \mathrm{~h}$ from the acute event, and subsequent expansion over the following $48 \mathrm{~h}$. MVO increases up to three times during the first 2 days, reaching a steady-state between day 2 and 9, that represent the best time to quantify MVO [18]. By reflecting extensive microvascular damage, MVO has been shown to provide important prognostic insights. There is a linear relation between MVO extent and scar size, not only in the acute but also in the chronic setting, as the acute MVO is a predictor of transmural extent at 6 months [19].
MVO is a powerful predictor of adverse cardiovascular outcome, also independent of left ventricular ejection fraction (LVEF) [8, 19-22]. On a cohort of 438 reperfused STEMI patients undergoing CMR within 3 days from the acute event, in contrast to early MVO, only late MVO independently predicted adverse events [23]. De Waha et al. also showed that the ratio $\mathrm{MVO} / \mathrm{scar}$ size is a more powerful predictor of adverse outcome then either considered alone [24]. Extravasation of erythrocytes into the myocardium as a consequence of disruption of capillaries and break-down of capillary barrier can be easily detected as a dark core area on T2-weighted images (Fig. 1c), due to the intrinsic paramagnetic properties of haemoglobin breakdown products, which shorten $\mathrm{T} 2$ relaxation time. Intramyocardial haemorrhage has been shown to be significantly related to infarct size and time to reperfusion, both in experimental models and in patients after percutaneous or surgical myocardial revascularization [15]. Out of 346 patients undergoing CMR after acute reperfused STEMI, Eitel et al. [25] found evidence of IMH in $35 \%$ of patients; in a multivariable model, infarct size, MVO extent and impaired LVEF were the strongest predictors of IMH. As expression of severe reperfusion injury, IMH has proved to have prognostic implications [25-27], demonstrating a strong unadjusted association with major adverse cardiovascular events [25, 27]. Intramyocardial haemorrhage is also a determinant of adverse left ventricular remodelling at 6 months follow-up; each myocardial segment showing IMH on T2-weighted images increases the risk of dilated left ventricular end-systolic volume by $50 \%$ [27].

\section{Complications of acute myocardial infarction}

With the onset of the reperfusion era, mechanical complications of acute MI, which are associated with reduced shortand long-term survival, have reduced to $<1 \%$ [28] (Fig. 2). The most encountered mechanical complications include ventricular free wall (Fig. 2a) or septal rupture, papillary muscle infarction (Fig. 2c) or rupture with secondary acute mitral regurgitation (MR), ventricular aneurysm and pseudo-aneurysm (Fig. 2b). With its superior spatial resolution and feasibility soon after the acute event, CMR is a promising tool for the identification of early and late MI complications [20]. Post MI ventricular septal defect (VSD) is associated with $80-90 \%$ mortality within few months after acute event; not only is CMR useful to make the diagnosis, but also tissue characterization to look for fibrosis of VSD edges helps identifying the most appropriate time for surgery [20]. Myocardial tissue characterization by CMR also allows to distinguish a true aneurysm, which is characterised by a large neck and typically enhanced aneurysm wall, from pseudo-aneurysm, which usually has a small 
Fig. 2 Complications of acute myocardial infarction. Two chamber long axis post-contrast sequence showing contained chronic rupture of the anterior wall (a, white arrow) in a patient with transmural myocardial infarction in the left anterior descending territory. Three chamber long axis cine sequence showing large pseudo-aneurysm of the mid-cavity inferolateral wall (b) with evidence of flow (b, white arrow-head) between a "tunnel-like" connection with the left ventricle in a patient with trasmural infarction in the left circumflex territory. Midcavity short-axis post-contrast sequence showing myocardial infarction of the papillary muscles (c). Mid-cavity short-axis post-contrast sequence showing myocardial infarction of the right ventricular inferior wall (d, black arrow) in a patient with transmural infarction in the basal inferior wall. Early (e) and late (f) four chamber long axis gadolinium enhancement sequences showing a large apical thrombus in a patient with transmural myocardial infarction in the distal left anterior descending territory (f)
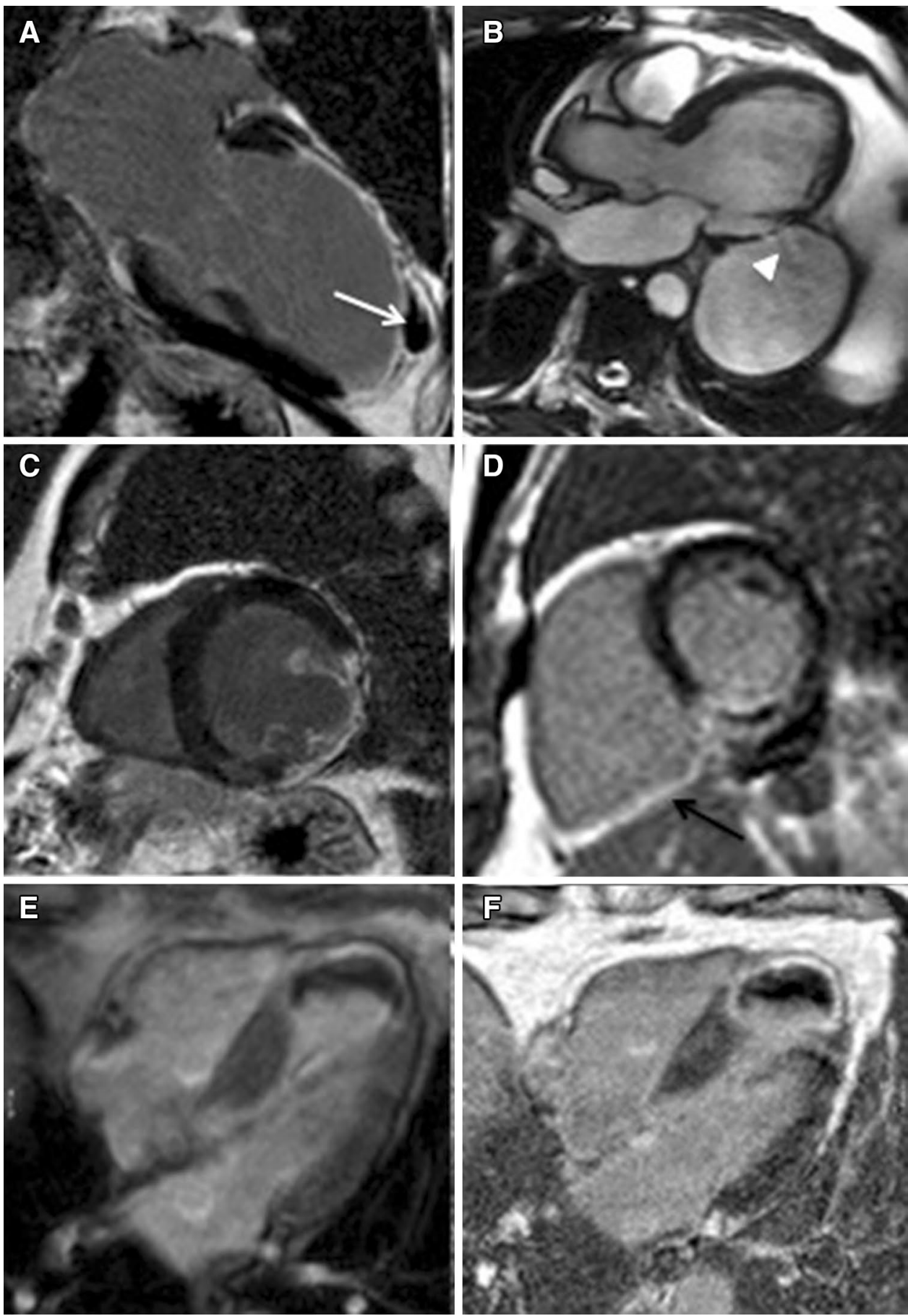

neck, with non-enhanced wall, and represents a contained rupture [20]. A lipomatous metaplasia seen as a high signal intensity area in the cine and post-contrast sequences, is also commonly seen in patients with old MI who underwent surgical myocardial revascularization [20]. The right ventricle $(\mathrm{RV})$ is involved in a non-negligible proportion of acute MI, mainly involving the inferior wall (incidence 24-50\%) [29], is haemodinamically relevant in up to $25-50 \%$ of cases and is usually associated with poorer prognosis [30, 31] (Fig. 2d). CMR has shown to detect RV involvement in $25 \%$ of patients with inferior MI, a percentage that is significantly superior to that detected by ECG and trans-thoracic echocardiogram (TTE). CMR studies have shown that RV myocardial infarction is usually associated with larger MI, lower LVEF and lower RV ejection fraction [30, 32]. LV thrombus is a frequent complication of ischemic heart disease, whose incidence increases with poor LVEF, greater scar size and ischemic aetiology [33] 
and whose diagnosis once relied on TTE. Given thrombus is avascular, it typically appears as a non-contrast gaining structure early (Fig. 2e) and late (Fig. 2f) after gadolinium administration on CMR; detection of thrombus by CMR has shown to be superior to TTE [34], also after contrast administration [35].

\section{Acute coronary syndromes with normal angiogram}

Up to a third of patients presenting with ACS shows normal arteries on coronary angiogram [12], making diagnosis and management challenging. Identifying a final diagnosis is important to guide patients' management as it has implications both on medical therapy (secondary prevention if it was a confirmed ACS) and prognosis [12]. A recent metaanalysis on patients with myocardial infarction and unobstructed coronaries reported in-hospital and 12 months allcause mortality of 0.9 and $4.7 \%$, respectively [36]. Among the differentials, the commonest causes of ACS with normal angiogram are acute myocarditis, MI secondary to distal embolization, coronary spasm or spontaneous recanalization and Tako-Tsubo cardiomyopathy (TTC). CMR plays a pivotal role in the diagnosis of these entities, mainly based on its higher spatial resolution and superior tissue characterization properties $[37,38]$. The unique selling point of CMR is indeed its ability to characterize myocardial tissue, based on the typical distribution pattern of the contrast agent, gadolinium: ischemic cardiomyopathy, following the ischemic wave-front, is typically characterized by late gadolinium enhancement (LGE) with a subendocardial to transmural distribution, while non-ischemic cardiomyopathy shows LGE with a mid-wall or epicardial distribution pattern, generally not located in the territory of distribution of a coronary artery [39]. A meta-analysis on more than 500 patients presenting with MI and unobstructed coronaries showed that one-third of patients had findings consistent with myocarditis [40] (Fig. 3a, b). Epicardial or mid-wall increased signal intensity on T2-weighted and early gadolinium enhancement sequences (Fig. 3a), with additional LGE in the post-contrast sequences (Fig. 3b), are the typical findings of acute myocarditis on CMR, which accurately resemble histologic Lake Louise criteria [41]. CMR is now recommended by European guidelines as first line imaging technique, prior to endomyocardial biopsy, in stable patients with suspected myocarditis [42]. Some studies suggest that myocarditis is a dynamic process which spreads from focal to disseminated myocardial involvement; CMR can timely follow-up this dynamic process [43]. The findings of LGE ischemic distribution pattern on CMR allows the identification of patients with "true" MI despite unobstructed coronaries on angiogram, such is the case of distal plaque embolization, coronary spasm or paradoxical embolization from a patent foramen ovale [38] (Fig. 3c, d). Tako-Tsubo cardiomyopathy, otherwise known as brokenheart syndrome or stress cardiomyopathy, accounts for up to $0.7-2.5 \%$ of all ACS, up to $12 \%$ in the female population [16] (Fig. 3e, f). TTC frequently presents as ACS-STEMI with evidence of unobstructed coronaries; it typically shows peculiar regional wall motion abnormality, involving the mid-distal segments of the heart. TTC is a reversible cardiomyopathy, which usually resolves within 6 months from the acute event, and can be detected on ventricular cine-angiogram and on TTE, mainly based on the typical regional wall motion abnormality. CMR provides an added value based on tissue characterization, showing a transient myocardial injury, characterised by the presence of myocardial oedema on T2-weighted images (Fig. 3e). Though it was once believed that in TTC LGE is absent, LGE has been found in a non-negligible proportion of cases, probably as a reflection of an underlying inflammatory response and transitory increase of extracellular space due to the myocardial oedema [44]. This is however reversible and no longer observed at follow-up in conjunction with the resolution of myocardial oedema/inflammation. The diagnostic value of CMR in patients with acute chest pain has been tested in the Emergency Department in the assessment of stable patients with chest pain, non-diagnostic electrocardiogram (ECG) and increased cardiac enzymes; in a study on 161 stable patients with acute chest pain, CMR showed high sensitivity and specificity in detecting ACS, being more sensitive and specific than the ECG [45].

\section{Chronic ischemic heart disease}

Due to its ability to identify myocardial fibrosis, CMR has had its widest application in the assessment of chronic ischemic heart disease (IHD) [46, 47]. Detection of fibrosis is based on the analysis of the distribution of the contrast agent within the myocardium, 10-15 min after its administration. CMR uses gadolinium-chelate contrast agent, an extra-cellular agent, which is quickly washed out by normal myocardium, and accumulates in damaged myocardium with expanded extra-cellular space [39]. Whether the extra-cellular space is expanded because of myocardial cell rupture in the setting of acute myocardial infarction or because of collagen deposition in the setting of chronic myocardial scarring, gadolinium will accumulate and be detected on CMR. Myocardial fibrosis can be present as infarct, replacement or diffuse fibrosis; these represent a continuum of myocardial damage, rather than isolated entities, as shown by Beltrami et al. [48] who found that replacement and diffuse fibrosis account for $70 \%$ of all fibrotic tissue in end-stage IHD, while infarct fibrosis only 
Fig. 3 Acute coronary syndromes with normal angiogram. Early gadolinium enhancement sequences showing extensive epicardial enhancement (a, black arrow) and corresponding late gadolinium (LGE) on the post-contrast sequences (b, black arrow) in a patient with acute myocarditis. T2-weighted images showing focal discrete oedema in the mid-cavity inferolateral wall (c, white arrow) with corresponding LGE on post-contrast sequences (d, white arrow) in a patient with embolic myocardial infarction in the mid left circumflex territory. T2-weighted sequences showing acute myocardial oedema in the apical segments (e) with no evidence of late gadolinium enhancement (LGE) on the post-contrast sequences (f) in a patient with Tako-Tsubo cardiomyopathy
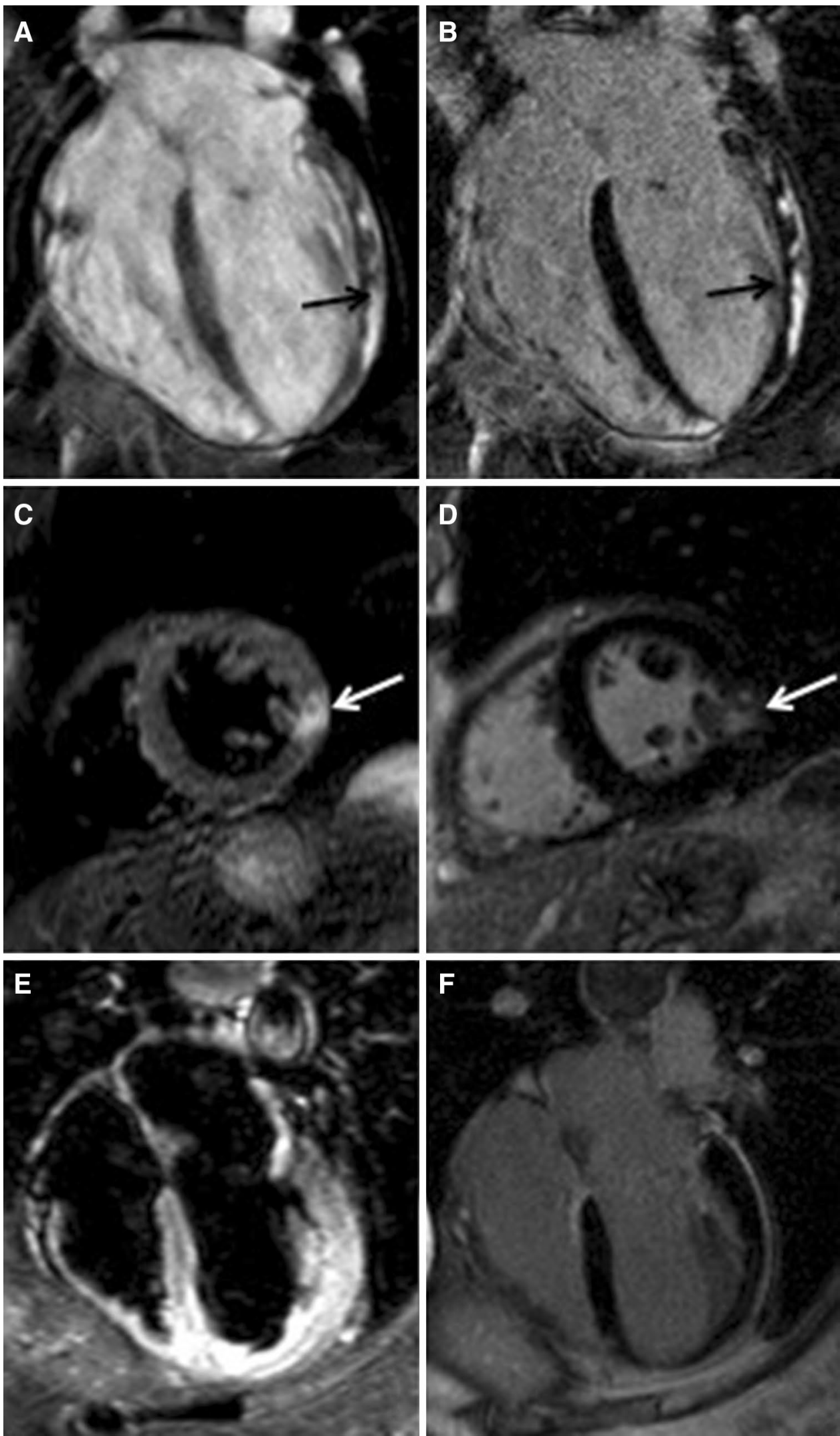
accounts for $30 \%$. The accuracy of CMR in assessing the ischemic scar has been validated in animal studies, which showed that the location, extent and shape of LGE on exvivo $\mathrm{CMR}$ were almost identical to the infarcted areas defined on histologic analysis [49]. CMR's excellent contrast and spatial resolution allow the identification of even small amounts of infarct scar, below $1 \mathrm{~g}$ of mass [50, 51]. CMR has shown to be superior to single photon emission tomography (SPECT) in the assessment of myocardial scar, especially in cases of small infarct and infarct in a nonanterior location [52]. Infarct healing is a well-known process, that can easily be detected and followed-up by CMR. A study on 58 reperfused STEMI patients showed that infarct size reduces 4 months after the acute event, but is relatively unchanged at 1 year follow-up [53]; in the acute phase infarct volume is influenced by hyperaemia, oedema and inflammation, which might explain the overestimation of infarct size early after the acute event [54]. However, infarct size at baseline has proved to be the strongest predictor of adverse long-term LV remodelling [53], which continues for up to a year after the acute event, also involving the remote non-infarcted myocardium. There is a strong linear relation between scar size, LV end-systolic (LVESV) and end-diastolic volumes (LVEDV) and LVEF. Scar size is the strongest predictor of LVEF, independent of scar location and transmurality [55]. A study on 90 reperfused anterior STEMI undergoing CMR early (3-5 days) and 90 days from the acute event showed that baseline infarct size, infarct heterogeneity and myocardial salvage are significantly associated with 90-day LVEF [56].

\section{Myocardial viability}

Transient ischemia impairs LV function, acutely and chronically, if repeated episodes occur; acute ischemic LV impairment is known as myocardial stunning, while chronic ischemic impairment is known as myocardial hibernation [57]. However, ischemic LV dysfunction is not necessarily an irreversible process, and LV function can improve after revascularization [58]. Myocardial viability is a reflection of impaired ischemia-induced contractility at rest that recovers after revascularization. The assessment of myocardial viability is the cornerstone to guide clinical treatment, as it has been shown that complete revascularization of viable myocardium reduces long-term adverse events, as compared to medical therapy [59]. Myocardial viability was once defined on TTE and PET, by means of LV wall thickness, wall motion abnormality on low-dose dobutamine echocardiography [57] and reduced metabolism. Low-dose dobutamine stress CMR has good specificity (83\%) and moderate sensitivity (74\%), not dissimilar from those of stress dobutamine echocardiography; a "bi-phasic response" is highly predictive of functional recovery [60]. The concept of myocardial viability was expanded by the implementation of scar analysis on CMR [61], which has shown better sensitivity, specificity and accuracy than SPECT in the prediction of myocardial viability [62]. Extent of LGE is significantly associated with myocardial viability and functional recovery. Kim et al. [63] assessed LGE extent and myocardial contractility before and after revascularization in 50 patients presenting with impaired LV systolic function, showing that improvement in LV contractility decreases with the increase in scar transmurality: $78 \%$ of LV dysfunctional segments with no LGE improved in function, compared to only $2 \%$ of those with evidence of $>75 \%$ LGE. A study on patients undergoing CMR before, early (6 days) and late (6 months) after surgical revascularization, showed that LGE transmurality strongly related to recovery in regional function at 6 months [64]. Using a $50 \%$ trasmural viability cut-off, 10 viable + normal segments predicted $\geq 3 \%$ improvement in LVEF, with a sensitivity of $95 \%$ and specificity of $75 \%$ [65]. Different studies found that irreversibly damaged myocardium was characterised by reduced wall thickness, so that reduced end-diastolic wall thickness was predictive of no myocardial recovery after revascularization [66]. Though regional LV wall thinning has been long thought to represent chronic transmural scar, Shah et al. [67] showed that among patients with CAD found to have $\mathrm{LV}$ wall thinning $(\leq 5.5 \mathrm{~mm}$ at end-diastole) $18 \%$ had only a small scar burden $(\leq 50 \%$ of total extent), which was associated with improved contractility and resolution of wall thinning after revascularization (Fig. 4). The assessment of scar in IHD has important prognostic implications. First, it predicts late myocardial recovery soon after the acute ischemic event; LGE volume after acute STEMI proved to be the strongest predictor of late LV dysfunction, over and above infarct transmurality, MVO and myocardial salvage, with a hazard ratio of 6.1 for adverse events when LGE extent was $\geq 23 \%$ [68]. The association of greater infarct size and impaired LVEF predicts even poorer outcome [69]. Infarct size, LVEF and LVESV on CMR predict future cardiac events early (1 week) after acute STEMI [70]. Among patients with clinically suspected CAD but with no history of MI, evidence of LGE on CMR is associated with worse outcome, and remains the strongest predictor of adverse events, even after adjustment for significant CAD on angiogram, LVEF and wall motion abnormality $[71,72]$.

\section{Risk stratification}

The assessment of infarct scar is important also to risk stratify patients according to their risk of arrhythmic events, as it is well known that myocardial scar represents 
Fig. 4 Myocardial viability and left ventricular (LV) recovery after revascularization. Longaxis two (a) and four chamber (b) cine sequences showing marked thinning of the LV anterior and anteroseptal walls in a patient with severe disease of the mid-distal left anterior descending (LAD); post-contrast sequences showing viable myocardium in the mid-distal LAD territory as late gadolinium enhancement (LGE) is limited to the subendocardium (c, d, white arrow). Longaxis two (e) and four chamber (f) cine sequences showing recovery of LV wall thickness 3 months after revascularization of the LAD
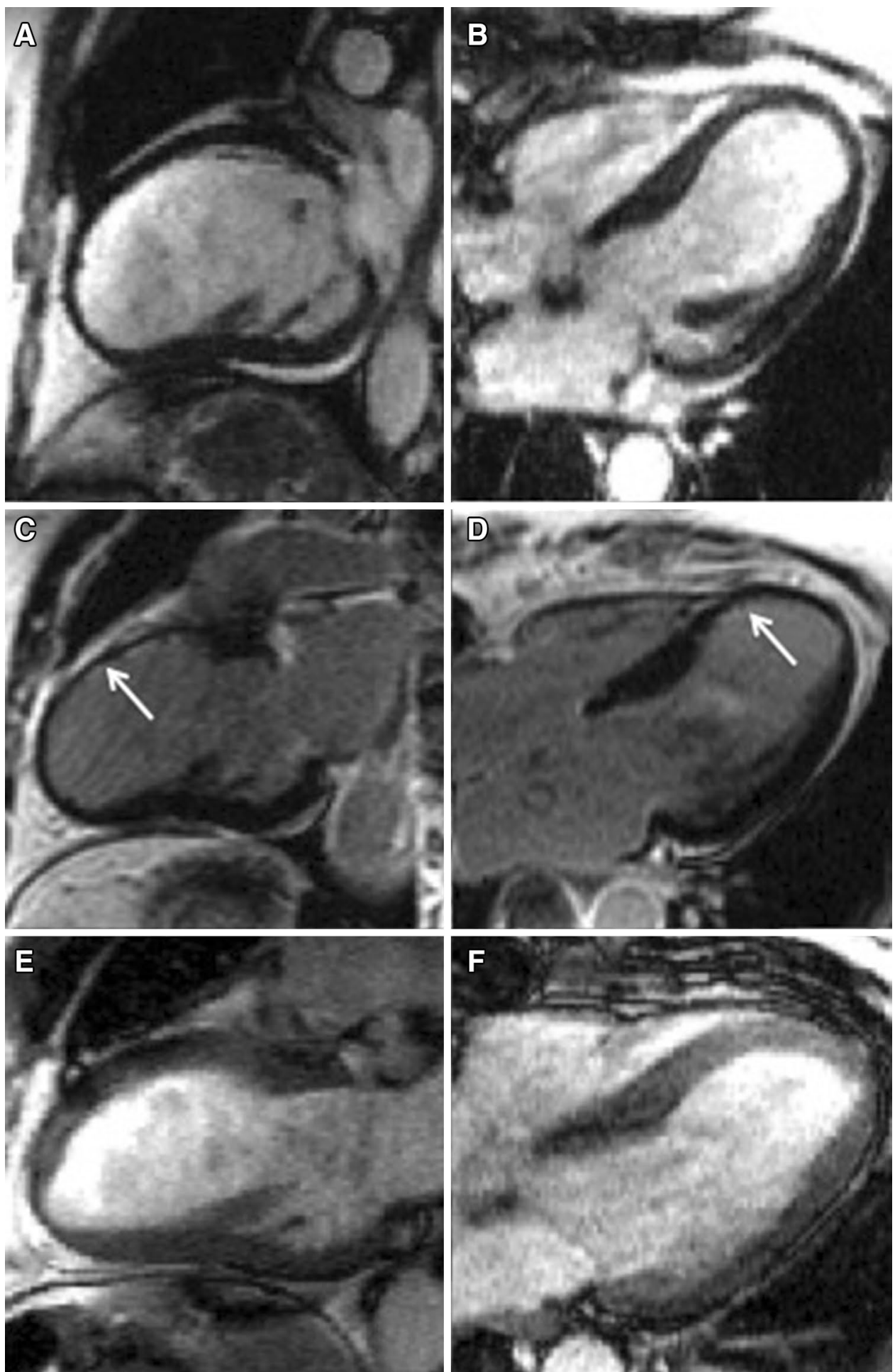

the substrate for re-entrant arrhythmias [73]. Different studies on patients undergoing CMR prior to ICD implantation have shown that scar extent is stronger than LVEF in predicting arrhythmic events (sudden death, ICD discharge, ventricular arrhythmias) and inducibility at electrophysiologic study (EPS) [74-76] and remains the strongest predictor, also in patients with preserved ejection fraction [77].
Scar analysis on CMR is based on different signal intensity of the infarcted area as compared to an area of normal, remote myocardium. Most studies have defined the cut-off for abnormal signal intensity at $5 \mathrm{SD}$ above that of normal myocardium to identify the core infarct and between 2 and 3 SD above normal myocardium to identify the peri-infarct zone, though the full width at half maximum technique has 
shown to be the most accurate and reproducible, regardless of the underlying myocardial disease [78]. Detailed semiautomated scar analysis allowed the identification of an even higher association between the peri-infarct zone and the arrhythmic risk. It is now believed that tissue heterogeneity is the strongest predictor of EPS inducibility and freedom from recurrence after ventricular tachycardia ablation $[79,80]$. CMR is thus a valuable tool also in the assessment of patients prior to ICD implantation, and there is recent evidence that scar extent on CMR predicts response to cardiac resynchronization therapy (CRT) [81, 82]; in a study on 47 IHD patients undergoing CRT, CMR showed that response rate to CRT was higher in patients with higher LVEF, smaller scar and lower number of LV segments with $>51 \%$ scar transmurality [82]. Finally, primary prevention ICD implantation is based on LVEF [83]. CMR is the gold standard for the assessment of LVEF as it is free from any geometric assumption $[84,85]$ : based on full 3D coverage of the heart, by contouring the endo- and epicardial borders, CMR provides 3D volumetric assessment of ventricular volumes and function.

\section{Stress perfusion CMR}

Stress CMR has been recently recognised as a reliable technique to diagnose myocardial ischemia in the setting of CAD. It is based on the assessment of myocardial perfusion during pharmacological stress testing with coronary vasodilators (Fig. 5), and on the detection of inducible wall motion abnormalities during high-dose dobutamine infusion (HDD-CMR). The most used vasodilator is adenosine, mainly because of its short-life and limited side effects; adenosine increases coronary artery vasodilatation in normal coronaries, but it does not increase blood flow downstream to stenotic arteries as the arteriolar bed is already maximally dilated (so-called "coronary steal" phenomenon); this allows the identification of areas of hypoperfused myocardium distal to a significant stenosis [86], which appear as a low signal intensity (dark) area on stress perfusion images (Fig. 5a-c). Stress perfusion CMR showed good diagnostic performances in several studies [87]. In a meta-analysis of 12 studies (761 patients) using fractional flow reserve (FFR) as a reference standard, perfusion CMR had a sensitivity of $89.1 \%$ and specificity of $84.9 \%$ on a patient basis as well as a sensitivity of $87.7 \%$ and specificity of $88.6 \%$ on a coronary territory basis [88]. Nandalur et al. showed that perfusion CMR has a sensitivity of $91 \%$ and a specificity of $81 \%$ in a per-patient analysis, and of 84 and $85 \%$ respectively for the identification of the ischemic segments [89]. Recently, a large randomized trial has shown that perfusion CMR has better sensitivity and negative predictive values compared to SPECT, and that it offers an accurate assessment of single-vessel and multivessel coronary disease, irrespective of the cut-off used for defining clinically significant coronary artery stenosis [90].

Perfusion CMR assessment of coronary flow reserve has also been compared with PET, and there was an excellent correlation between the two modalities [91]. Dobutamine is a sympatomimetic amine with positive inotropic and chronotropic effects, that mimics the physiological effect of physical exercising, inducing increased oxygen demand [86]. When administered at low-dose it usually determines the recruitment of the contractile reserve of hibernating myocardial segments (dysfunctional but viable myocardium at rest), but when given at high-dose it induces ischemia in territories with significant coronary artery stenosis, which is expressed as the onset of wall motion abnormalities, easy to recognize on CMR cine images. HDD-CMR has been shown to be superior to dobutamine stress echo (DSE) with significantly higher diagnostic accuracy, probably due to superior image quality allowing a better assessment of regional wall motion abnormalities [92]. However "the visual assessment-based" interpretation of wall motion abnormalities can lead to different results. Recently, the introduction of the feature tracking software (FT), allowing the assessment of myocardial strain from cine images, without the need for additional sequences, has improved this limitation. Schneeweis et al. showed that FT based analysis of circumferential strain during HDD-CMR was feasible and helped differentiating between normal myocardium and segments supplied by a stenotic coronary artery, suggesting that the quantitative assessment of myocardial strain with FT may improve the diagnostic accuracy of HDD-CMR for detection of ischemia [93].

\section{Prognosis}

Many studies have been published regarding the assessment of prognosis with stress-CMR [94, 95]. Sozzi et al., showed that among 326 consecutive patients with normal adenosine stress perfusion CMR the event rate was low and prognosis excellent over a 5.5-year period [96]. Kelle et al. found that, in a large cohort of patients with negative dobutamine stress, the annual cardiac event rate was $1.1 \%$ while the hazard ratio associated with a positive test was 3.3 [97]. In a large meta-analysis of 19 studies, involving a total of 11,636 patients with a mean follow up of 32 months, Lipinski et al. found that a negative stress CMR was associated with very low-risk of cardiovascular death and myocardial infarction, suggesting that stress CMR may help in the risk stratification of patients with known or suspected CAD [98]. Based on those data, current clinical guidelines for myocardial revascularization, suggest the use of stress 

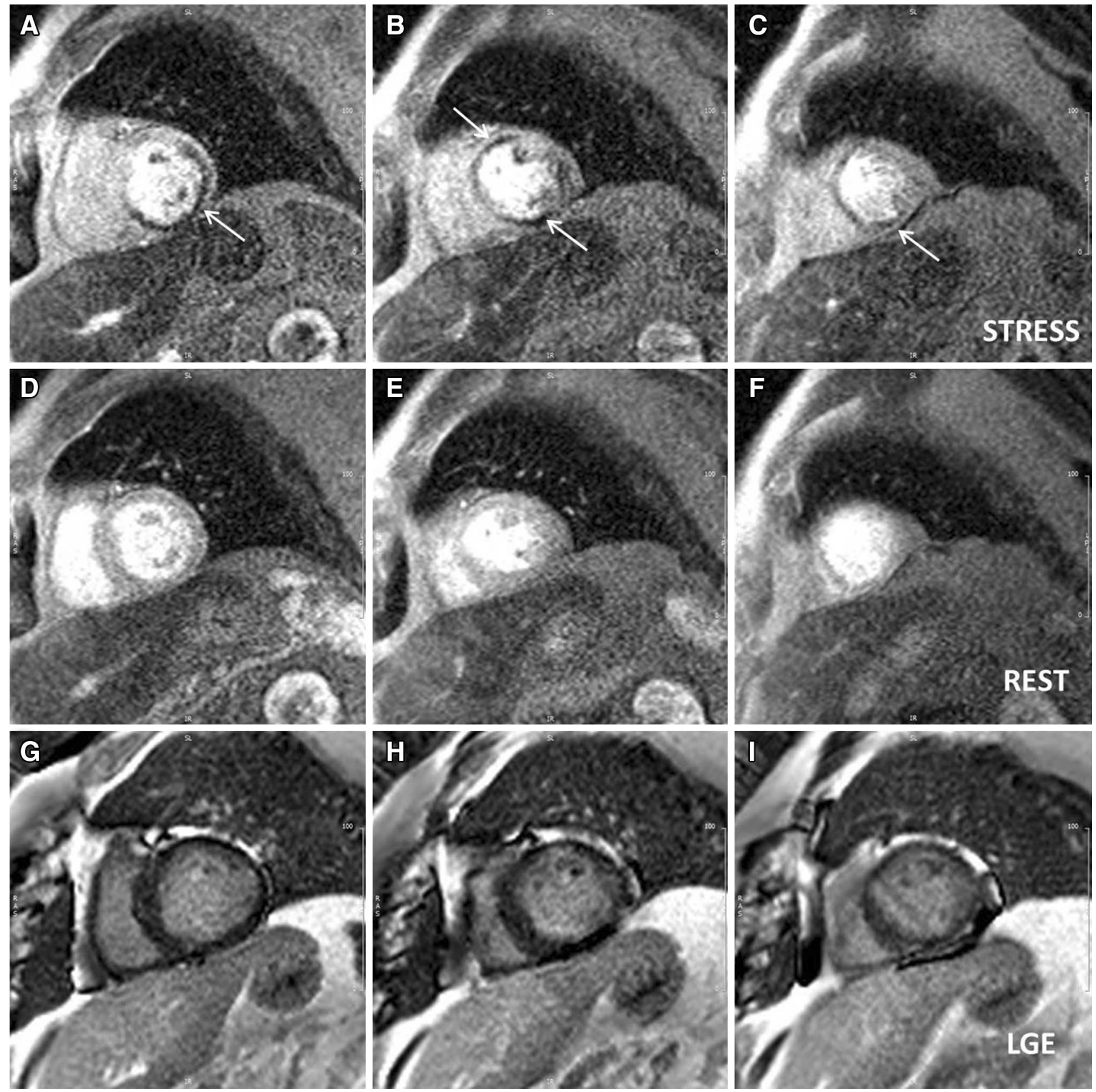

Fig. 5 Stress perfusion CMR. Three slice (base, mid and apex-a, $\mathbf{b}$ and $\mathbf{c}$, respectively) short axis stress perfusion sequences acquired at peak adenosine infusion showing two separate areas of hypoperfusion, in the proximal to distal inferior (a-c, white arrow) and midcavity anterior walls (b, white arrow), with a normal rest perfusion (d-f) and only a discrete area of subendocardial late gadolinium enhancement in the mid-cavity anterior wall (h) on post-contrast images $(\mathbf{g}-\mathbf{i})$. Overall, these findings are consistent with inducible myocardial ischemia in all right coronary artery territory with ischemia superimposed to the infarcted area in the mid left anterior descending territory
CMR in the management of patients with intermediate risk of CAD and stable symptoms (class IA) [99].

The 5-year follow up of the CE-MARC study demonstrated that compared to SPECT, CMR is the strongest predictor or risk for MACE, independent of cardiovascular risk factors, angiographic result, or initial patient's treatment [100]. 


\section{Indications of CMR in ischemic heart disease: international guidelines}

According to international guidelines, CMR plays a role in the assessment of patients with ischemic heart disease. 2013 ESC guidelines on stable angina recommended imaging stress testing (IB) for risk stratification in patients with known stable CAD and a deterioration of symptoms as a guide to clinical decision making and in the assessment of patients with intermediate pre-test probability (15-85\%) of CAD [101]. Imaging stress test was also recommended in symptomatic patients with prior myocardial revascularization or to assess functional severity of intermediate lesions on angiography (IIaB). Stress testing with CMR is also recommended before or after discharge to assess residual myocardial ischemia and viability in STEMI patients (IA) [1]. More recently, stress CMR has been given a class IA indication by the new European Society of Cardiology guidelines on myocardial revascularization [99] to assess suspected $\mathrm{CAD}$ in patients with intermediate pre-test probability.

\section{Limitations}

Though CMR is increasingly used, its availability is still limited in certain centres. CMR can definitely be performed safely also in the acute setting, but the patient needs to be haemodinamically stable. There are few contraindications to CMR. Patients with non-MR conditional devices (intracranial clips, neuro-stimulator, metallic objects in the eye) should not be offered a CMR; recent advances in technology do provide MR-conditional cardiac devices (pace-maker and ICD), that allow CMR scanning, though under strict medical monitoring. Gadolinium-chelate contrast agent is safer than iodine contrast agents, but should be avoided in severe renal dysfunction (eGFR $<30 \mathrm{ml} /$ $\min / 1.73 \mathrm{~m}^{2}$ ), as it increases the risk of nephrogenic systemic fibrosis, a potentially fatal condition. Claustrophobia has long been thought to be an absolute contraindication to CMR; however, performing CMR with the patient lying prone, providing an angulated mirror in the CMR bore to allow the patients to look outside the scanner and inviting a relative to sit at the end of scanner should help reduce these cases to a negligible percentage.

\section{Conclusion}

CMR is a well-established imaging tool, which allows a comprehensive, multi-parametric cardiac assessment in a 40-min one-stop-shop technique. The use of CMR in ischemic heart disease has rapidly spread given its superior diagnostic properties and important prognostic implications.

\section{Compliance with ethical standards}

Conflict of interest C Bucciarelli-Ducci is consultant for Circle Cardiovascular Imaging.

Open Access This article is distributed under the terms of the Creative Commons Attribution 4.0 International License (http:// creativecommons.org/licenses/by/4.0/), which permits unrestricted use, distribution, and reproduction in any medium, provided you give appropriate credit to the original author(s) and the source, provide a link to the Creative Commons license, and indicate if changes were made.

\section{References}

1. Steg PG, James SK, Atar D et al (2012) ESC guidelines for the management of acute myocardial infarction in patients presenting with ST-segment elevation. Eur Heart J 33(20):2569-2619

2. Abdel-Aty H, Simonetti O, Friedrich MG (2007) T2-weighted cardiovascular magnetic resonance imaging. J Magn Reson Imaging 26:452-459

3. Ridgway JP (2010) Cardiovascular magnetic resonance physics for clinicians: part I. J Cardiovasc Magn Reson 12(1):71

4. Biglands JD, Radjenovic A, Ridgway JP (2012) Cardiovascular magnetic resonance physics for clinicians: part II. J Cardiovasc Magn Reson 14(1):66

5. Eitel I, Friedrich MG (2011) T2-weighted cardiovascular magnetic resonance in acute cardiac disease. J Cardiovasc Magn Reson 13(1): 13

6. Francone M, Bucciarelli-Ducci C, Carbone I et al (2009) Impact of primary coronary angioplasty delay on myocardial salvage, infarct size, and microvascular damage in patients with ST-segment elevation myocardial infarction: insight fro cardiovascular magnetic resonance. JACC 54(23):2145-2153

7. Aletras AH, Tilak GS, Natanzon A et al (2006) Retrospective determination of the area at risk for reperfused acute myocardial infarction with $\mathrm{T} 2$-weighted cardiac magnetic resonance imaging: histopathological and displacement encoding with stimulated echoes (DENSE) functional validations. Circulation 113(15):1865-1870

8. Desch S, Eitel I, de Waha S et al (2011) Cardiac magnetic resonance imaging parameters as surrogate endpoints in clinical trials of acute myocardial infarction. Trials 12(1):204

9. Rajiah P, Deasi MY, Kwon D, Flamm SD (2013) MR imaging of myocardial infarction. Radiographics 33:1383-1412

10. Kerensky RA, Wade M, Deedwania P et al (2002) Revisiting the culprit lesion in non-Q-wave myocardial infarction: results from the VANQWISH trial angiographic core laboratory. J Am Coll Cardiol 39(9):1456-1463

11. Monney PA, Sekhri N, Burchell T et al (2011) Acute myocarditis presenting as acute coronary syndrome: role of early cardiac magnetic resonance in its diagnosis. Heart 97(16):1312-1319

12. Gallagher S, Jones DA, Anand V, Mohiddin S (2012) Diagnosis and management of patients with acute cardiac symptoms, troponin elevation and culprit-free angiograms. Heart 98(13):974-982

13. Raman SV, Simonetti OP, Winner MW et al (2010) Cardiac magnetic resonance with edema imaging identifies myocardium at risk and predicts worse outcome in patients with 
non-ST-segment elevation acute coronary syndrome. J Am Coll Cardiol 55(22):2480-2488

14. Walls MC, Verhaert D, Raman SV (2011) Myocardial edema imaging in acute coronary syndromes. J Magn Reson Imaging 34(6):1243-1250

15. Basso C, Thiene G (2006) The pathophysiology of myocardial reperfusion: a pathologist's perspective. Heart 92(11):1559-1562

16. Hunold P, Bischoff P, Barkhausen J, Vogt FM (2012) Acute chest pain: the role of MR imaging and MR angiography. Eur J Radiol 81(12):3680-3690

17. Rochitte CE, Lima AC, Bluemke DA et al (1998) Magnitude and time course of microvascular obstruction and tissue injury after acute myocardial infarction. Circulation 98(10):1006-1015

18. Beek AM, van Rossum AC (2010) Cardiovascular magnetic resonance imaging in patients with acute myocardial infarction. Heart 96:237-243

19. Wu KC, Zerhouni EA, Judd RM et al (1998) Prognostic significance of microvascular obstruction by magnetic resonance imaging in patients with acute myocardial infarction. Circulation 97(8):765-772

20. Flavian A, Carta F, Thuny F et al (2012) Cardiac MRI in the diagnosis of complications of myocardial infarction. Diagn Interv Imaging 93(7-8):578-585

21. Eitel I, de Waha, Wöhrle J et al (2014) Comprehensive prognosis assessment by CMR imaging after ST-segment elevation myocardial infarction. J Am Coll Cardiol 64(12):1217-1226

22. Hombach V, Grebe O, Merkle N et al (2005) Sequelae of acute myocardial infarction regarding cardiac structure and function and their prognostic significance as assessed by magnetic resonance imaging. Eur Heart J 26(6):549-557

23. de Waha S, Desch S, Eitel I et al (2010) Impact of early vs. late microvascular obstruction assessed by magnetic resonance imaging on long-term outcome after ST-elevation myocardial infarction: a comparison with traditional prognostic markers. Eur Heart J 31(21):2660-2668

24. de Waha S, Desch S, Eitel I et al (2012) Relationship and prognostic value of microvascular obstruction and infarct size in STelevation myocardial infarction as visualized by magnetic resonance imaging. Clin Res Cardiol 101(6):487-495

25. Eitel I, Kubusch K, Strohm O et al (2011) Prognostic value and determinants of a hypointense infarct core in T2-weighted cardiac magnetic resonance in acute reperfused ST-elevation-myocardial infarction. Circ Cardiovasc Imaging 4(4):354-363

26. Romero J, Lupercio F, Carlos J et al (2016) Microvascular obstruction detected by cardiac MRI after AMI for the prediction of LV remodeling and MACE: a meta-analysis of prospective trials. Int J Cardiol 202:344-348

27. Husser O, Monmeneu JV, Sanchis J et al (2013) Cardiovascular magnetic resonance-derived intramyocardial hemorrhage after STEMI: influence on long-term prognosis, adverse left ventricular remodeling and relationship with microvascular obstruction. Int J Cardiol 167(5):2047-2054

28. Kutty RS, Jones N, Moorjani N (2013) Mechanical complications of acute myocardial infarction. Cardiol Clin 31(4):519-531

29. Ondrus T, Kanovsky J, Novotny T et al (2013) Right ventricular myocardial infarction: from pathophysiology to prognosis. Exp Clin Cardiol 18(1):27-30

30. Jensen CJ, Jochims M, Hunold P et al (2010) Right ventricular involvement in acute left ventricular myocardial infarction: prognostic implications of MRI findings. Am J Roentgenol 194(3):592-598

31. Larose E, Ganz P, Reynolds HG et al (2007) Right ventricular dysfunction assessed by cardiovascular magnetic resonance imaging predicts poor prognosis late after myocardial infarction. J Am Coll Cardiol 49(8):855-862

32. Kim HW, Farzaneh-far A, Kim RJ (2010) Cardiovascular magnetic resonance in patients with myocardial infarction current and emerging applications. J Am Coll Cardiol 55(1):1-16

33. Weinsaft JW, Kim HW, Shah DJ et al (2008) Detection of left ventricular thrombus by delayed-enhancement cardiovascular magnetic resonance prevalence and markers in patients with systolic dysfunction. J Am Coll Cardiol 52(2):148-157

34. Mollet NR, Dymarkowski S, Volders W et al (2002) Visualization of ventricular thrombi with contrast-enhanced magnetic resonance imaging in patients with ischemic heart disease. Circulation 106(23):2873-2877

35. Weinsaft JW, Kim RJ, Ross M et al (2009) Contrast-enhanced anatomic imaging as compared to contrast-enhanced tissue characterization for detection of left ventricular thrombus. JACC Cardiovasc Imaging 2(8):969-979

36. Pasupathy S, Air T, Dreyer RP, Tavella R, Beltrame JF (2015) Systematic review of patients presenting with suspected myocardial infarction and nonobstructive coronary arteries. Circulation 131(10):861-870

37. Assomull RG, Lyne JC, Keenan N et al (2007) The role of cardiovascular magnetic resonance in patients presenting with chest pain, raised troponin, and unobstructed coronary arteries. Eur Heart J 28(10):1242-1249

38. Dastidar AG, Rodrigues JCL, Ahmed N, Baritussio A, Bucciarelli-Ducci C (2015) The role of cardiac MRI in patients with troponin-positive chest pain and unobstructed coronary arteries. Curr Cardiovasc Imaging Rep 8(8):28

39. Mahrholdt H, Wagner A, Judd RM, Sechtem U, Kim RJ (2005) Delayed enhancement cardiovascular magnetic resonance assessment of non-ischaemic cardiomyopathies. Eur Heart $\mathbf{J}$ 26(15):1461-1474

40. Tornvall P, Gerbaud E, Behaghel A et al (2015) Myocarditis or "true" infarction by cardiac magnetic resonance in patients with a clinical diagnosis of myocardial infarction without obstructive coronary disease: a meta-analysis of individual patient data. Atherosclerosis 241(1):87-91

41. Laissy JP, Hyafil F, Feldman LJ et al (2005) Differentiating acute myocardial infarction from myocarditis: diagnostic value of early- and delayed-perfusion cardiac MR imaging. Radiology 237(1):75-82

42. Caforio ALP, Pankuweit S, Arbustini E et al (2013) Current state of knowledge on aetiology, diagnosis, management, and therapy of myocarditis: a position statement of the European Society of Cardiology Working Group on Myocardial and Pericardial Diseases. Eur Heart J 34:2636-2648

43. Friedrich MG, Strohm O, Schulz-Menger J et al (1998) Contrast media-enhanced magnetic resonance imaging visualizes myocardial changes in the course of viral myocarditis. Circulation 97(18):1802-1809

44. Dastidar AG, Frontera A, Palazzuoli A, Bucciarelli-Ducci C (2015) TakoTsubo cardiomyopathy: unravelling the malignant consequences of a benign disease with cardiac magnetic resonance. Heart Fail Rev 20:415-421

45. Kwong RY, Schussheim AE, Rekhraj S et al (2003) Detecting acute coronary syndrome in the emergency department with cardiac magnetic resonance imaging. Circulation 107(4):531-537

46. Florian A, Jurcut R, Ginghina C, Bogaert J (2011) Cardiac magnetic resonance imaging in ischemic heart disease: a clinical review. J Med Life 4(4):330-345

47. Doesch C, Papavassiliu T (2014) Diagnosis and management of ischemic cardiomyopathy: role of cardiovascular magnetic resonance imaging. World J Cardiol 6(11):1166 
48. Beltrami CA, Finato N, Rocco M et al (1994) Structural basis of end-stage failure in ischemic cardiomyopathy in humans. Circulation 89(1):151-163

49. Kim RJ, Fieno DS, Parrish TB et al (1999) Relationship of MRI dealyed contrast enhancement to irreversible injury, infarct age, and contractile function. Circulation 100:1992-2002

50. Schwitter J, Arai AE (2011) Assessment of cardiac ischaemia and viability: role of cardiovascular magnetic resonance. Eur Heart J 32(7):799-809

51. Karamitsos TD, Dall'Armellina E, Choudhury RP, Neubauer S (2011) Ischemic heart disease: comprehensive evaluation by cardiovascular magnetic resonance. Am Heart J 162(1):16-30

52. Ibrahim T, Bülow HP, Hackl T et al (2007) Diagnostic value of contrast-enhanced magnetic resonance imaging and single-photon emission computed tomography for detection of myocardial necrosis early after acute myocardial infarction. J Am Coll Cardiol 49(2):208-216

53. Ganame J, Messalli G, Masci PG et al (2011) Time course of infarct healing and left ventricular remodelling in patients with reperfused ST segment elevation myocardial infarction using comprehensive magnetic resonance imaging. Eur Radiol 21(4):693-701

54. Masci PG, Bogaert J (2012) Post myocardial infarction of the left ventricle: the course ahead seen by cardiac MRI. Cardiovasc Diagn Ther 2(2):113-127

55. Orn S, Manhenke C, Anand IS et al (2007) Effect of left ventricular size, location, and transmurality on left ventricular remodeling with healed myocardial infarction. Am J Cardiol 99(8):1109-1114

56. Grover S, Bell G, Lincoff M et al (2015) Utility of CMR markers of myocardial injury in predicting LV functional recovery: results from PROTECTION AMI CMR Sub-study. Heart Lung Circ 24(9):891-897

57. Camici PG, Prasad SK, Rimoldi OE (2008) Stunning, hibernation and assessment of myocardial viability. Circulation 117:103-114

58. Birnbaum Y, Kloner RA (1996) Myocardial viability. West J Med 165(6):364-371

59. Gerber BL, Rousseau MF, Ahn SA et al (2012) Prognostic value of myocardial viability by delayed-enhanced magnetic resonance in patients with coronary artery disease and low ejection fraction impact of revascularization therapy. J Am Coll Cardiol 59(9):825-835

60. Kaandorp TAM, Lamb HJ, van der Wall EE, de Roos A, Bax JJ (2005) Cardiovascular MR to assess myocardial viability in chronic ischaemic LV dysfunction. Heart 91:1359-1365

61. Jerosh-Herold M, Kwong RJ (2008) Magnetic resonance imaging in the assessment of ventricular remodeling and viability. Curr Heart Fail Rep 5(1):5-10

62. Kitagawa K, Sakuma H, Hirano T et al (2003) Acute myocardial infarction: myocardial viability assessment in patients early thereafter-comparison of contrast-enhanced MR imaging with resting (201)Tl SPECT: single photon emission computed tomography. Radiology 226(1):138-144

63. Kim RJ, Wu E, Rafael A et al (2000) The use of contrastenhanced magnetic resonance imaging to identify reversible myocardial dysfunction. N Engl J Med 343:1445-1453

64. Selvanayagam JB, Kardos A, Francis JM et al (2004) Value of delayed-enhancement cardiovascular magnetic resonance imaging in predicting myocardial viability after surgical revascularization. Circulation 110:1535-1541

65. Pegg TJ, Selvanayagam JB, Jennifer J et al (2010) Prediction of global left ventricular functional recovery in patients with heart failure undergoing surgical revascularisation, based on late gadolinium enhancement cardiovascular magnetic resonance. J Cardiovasc Magn Reson 12(1):56
66. Baer FM, Voth E, Schneidr CA et al (1995) Comparison of lowdose dobutamine-gradient-echo magnetic resonance imaging and positron emission tomography with $[18 \mathrm{~F}]$ fluorodeoxyglucose in patients with chronic coronary artery disease. A functional and morphological approach to the detection of residual myocardial viability. Circulation 91:1006-1015

67. Shah DJ, Kim HW, James O et al (2013) Prevalence of regional myocardial thinning and relationship with myocardial scarring in patients with coronary artery disease. JAMA 309(9):909-918

68. Larose E, Rodes-Cabau J, Pibarot P et al (2010) Predicting late myocardial recovery and outcomes in the early hours of ST-segment elevation myocardial infarction and necrosis characteristics by cardiovascular magnetic resonance. J Am Coll Cardiol 55(22):2459-2469

69. Izquierdo M, Ruiz-Granell R, Bonanad C et al (2013) Value of early cardiovascular magnetic resonance for the prediction of adverse arrhythmic cardiac events after a first noncomplicated ST-segment-elevation myocardial infarction. Circ Cardiovasc Imaging 6(5):755-761

70. Wu E, Ortiz JT, Tejedor P et al (2008) Infarct size by contrast enhanced cardiac magnetic resonance is a stronger predictor of outcomes than left ventricular ejection fraction or end-systolic volume index: prospective cohort study. Heart 94(6):730-737

71. Abbasi SA, Ertel A, Shah RV et al (2013) Impact of cardiovascular magnetic resonance on management and clinical decisionmaking in heart failure patients. J Cardiovasc Magn Reson 15(1):89

72. Catalano O, Moro G, Perotti M et al (2012) Late gadolinium enhancement by cardiovascular magnetic resonance is complementary to left ventricle ejection fraction in predicting prognosis of patients with stable coronary artery disease. J Cardiovasc Magn Reson 14:29

73. Scott PA, Rosengarten JA, Curzen NP, Morgan JM (2013) Late gadolinium enhancement cardiac magnetic resonance imaging for the prediction of ventricular tachyarrhythmic events: a metaanalysis. Eur J Heart Fail 15:1019-1027

74. Boyé P, Abdel-Aty H, Zacharzowsky U et al (2011) Prediction of life-threatening arrhythmic events in patients with chronic myocardial infarction by contrast-enhanced CMR. JACC Cardiovasc Imaging 4(8):871-879

75. Arenal A, Hernandez J, Perez-David E et al (2012) Do the spatial characteristics of myocardial scar tissue determine the risk of ventricular arrhythmias? Cardiovasc Res 94(2):324-332

76. Rijnierse MT, Allaart CP, de Haan S et al (2016) Non-invasive imaging to identify susceptibility for ventricular arrhythmias in ischaemic left ventricular dysfunction. Heart 102:832-840

77. Yalin K, Golcuk E, Buyukbayrak H et al (2014) Infarct characteristics by CMR identifies substrate for monomorphic VT in post-MI patients with relatively preserved systolic function and ns-VT. PACE 37(4):447-453

78. Flett AS, Hasleton J, Cook C et al (2011) Evaluation of techniques for the quantification of myocardial scar of differing etiology using cardiac magnetic resonance. JACC Cardiovasc Imaging 4(2):150-156

79. Schmidt A, Azevedo CF, Cheng A et al (2007) Infarct tissue heterogeneity by magnetic resonance imaging identifies enhanced cardiac arrhythmia susceptibility in patients with left ventricular dysfunction. Circulation 115(15):2006-2014

80. Estner HL, Zviman MM, Herzka D et al (2011) The critical isthmus sites of ischemic ventricular tachycardia are in zones of tissue heterogeneity, visualized by magnetic resonance imaging. Heart Rhythm 8(12):1942-1949

81. White JA, Yee R, Yuan X et al (2006) Delayed enhancement magnetic resonance imaging predicts response to cardiac resynchronization therapy in patients with intraventricular dyssynchrony. J Am Coll Cardiol 48(10):0-7 
82. Chalil S, Foley PW, Muyhaldeen SA et al (2007) Late gadolinium enhancement-cardiovascular magnetic resonance as a predictor of response to cardiac resynchronization therapy in patients with ischaemic cardiomyopathy. Europace 9(11):1031-1037

83. Brignole M, Auricchio A, Baron-Esquivias G et al (2013) ESC Guidelines on cardiac pacing and cardiac resynchronization therapy: the task force on cardiac pacing and resynchronization therapy of the European Society of Cardiology (ESC). Developed in collaboration with the European Heart Rhythm Association. Eur Heart J 34(29):2281-2329

84. Pennell DJ (2002) Ventricular volume and mass by CMR. J Cardiovasc Magn Reson 4(4):507-513

85. Pennell DJ (2010) Cardiovascular magnetic resonance. Circulation 121(5):692-705

86. Bettencourt N, Chiribiri A, Schuster A, Nagel E (2009) Assessment of myocardial ischemia and viability using cardiac magnetic resonance. Curr Heart Failr Rep 6(3):142-153

87. Schwitter J, Wacker CM, Van Rossum AC et al (2008) MRIMPACT: comparison of perfusion-cardiac magnetic resonance with single-photon emission computed tomography for the detection of coronary artery disease in a multicentre, multivendor, randomized trial. Eur Heart J 29(4):480-489

88. Desai RR, Jha S (2013) Diagnostic performance of cardiac stress perfusion MRI in the detection of coronary artery disease using fractional flow reserve as teh refernec standard: a metaanalysis. Am J Roentgenol 201(2):245-252

89. Nandalur KR, Dwamena BA, Choudhri AF, Nadalur MR, Carlos RC (2007) Diagnostic performance of stress cardiac magnetic resonance imaging in the detection of coronary artery disease: a meta-analysis. J Am Coll Cardiol 50(14):1343-1353

90. Greenwood JP, Maredia N, Younger JF et al (2012) Cardiovascular magnetic resonance and single-photon emission compute tomography for diagnosis of coronary artery disease (CEMARC): a prospective trial. Lancet 379:453-460

91. Schwitter J, Nanz D, Kneifel S et al (2001) Assessment of myocardial perfusion in coronary artery disease by magnetic resonance. A comparison with positron emission tomography and coronary angiography. Circulation 103:2230-2235

92. Nagel E, Lehmkuhl HB, Bocksch W et al (1999) Noninvasive diagnosis of ischemia-induced wall motion abnormalities with the use of high-dose dobutamine stress MRI. Comparison with dobutamine stress echocardiography. Circulation 99:763-770

93. Schneeweis C, Qiu J, Schnackenburg B et al (2014) Value of additional strain analysis with feature tracking in dobutamine stress cardiovascular magnetic resonance for detecting coronary artery disease. J cardiovasc Magn Reson 16:72

94. Ingkanisorn WP, Kwong RY, Bohme NS et al (2006) Prognosis of negative adenosine stress magnetic resonance in patients presenting to an emergency department with chest pain. J Am Coll Cardiol 47:1427-1432

95. Jahnke C, Nagel E, Gebker R et al (2007) Prognostic value of cardiac magnetic resonance stress test. Adenosine stress perfusion and dobutamine stress wall motion imaging. Circulation 115:1769-1776

96. Sozzi FB, Iacuzio L, Civaia F et al (2015) I Incremental value of normal adenosine perfusion cardiac magnetic resonance: long-term outcome. Am Heart J 169(6):841-846

97. Kelle S, Chiribiri A, Vierecke J et al (2011) Long-term prognostic value of stress CMR. JACC Cardiovasc Imaging 4(2):161-172

98. Lipinski MJ, McVey CM, Berger JS et al (2013) Prognostic value of stress cardiac magnetic resonance imaging in patients with known or suspected coronary artery disease: a systematic review and meta-analysis. J Am Coll Cardiol 62(9):826-838

99. Windecker S, Kohl P, Alfonso F et al (2014) ESC/EACTS guidelines on myocardial revascularization the task force on myocardial revascularization of the European Society of Cardiology (ESC) and the European Association. Eur Heart J 35:2541-2619

100. Greenwood JP, Herzog BA, Brown JM, et al (2016) Prognostic value of cardiovascular magnetic resonance and singlephoton emission computed tomography in suspected coronary heart disease: long-term follow-up of a prospective, diagnostic accuracy cohort study. Ann Intern Med. 165:1-9. doi:10.7326/ M15-1801

101. Montalescot G, Sechtem U, Achenbach S et al (2013) ESC guidelines on the management of stable coronary artery disease: the task force on the management of stable coronary artery disease of the European Society of Cardiology. Eur Heart J 34(38):2949-3003 